\title{
COMULTIPLICATIVITY OF THE OZSVÁTH-SZABÓ CONTACT INVARIANT
}

\author{
JoHN A. BALDWIN
}

\begin{abstract}
Suppose that $S$ is a surface with boundary and that $g$ and $h$ are diffeomorphisms of $S$ which restrict to the identity on the boundary. Let $Y_{g}, Y_{h}$, and $Y_{h g}$ be the 3-manifolds with open book decompositions given by $(S, g),(S, h)$, and $(S, h g)$, respectively. We show that the Ozsváth-Szabó contact invariant is natural under a comultiplication map $\tilde{\mu}: \widehat{H F}\left(-Y_{h g}\right) \rightarrow \widehat{H F}\left(-Y_{g}\right) \otimes \widehat{H F}\left(-Y_{h}\right)$. It follows that if the contact invariants associated to the open books $(S, g)$ and $(S, h)$ are non-zero then the contact invariant associated to the open book $(S, h g)$ is also non-zero. We extend this comultiplication to a map on $\mathrm{HF}^{+}\left(-Y_{h g}\right)$, and as a result we obtain obstructions to the 3-manifold $Y_{h g}$ being an $L$-space. We also use this to find restrictions on contact structures which are compatible with planar open books.
\end{abstract}

\section{Introduction}

In 2002, Ozsváth and Szabó discovered an invariant of contact 3-manifolds constructed as follows [12]. Given a contact 3-manifold $(Y, \xi)$, we can find a compatible (in the sense of Giroux [6]) open book decomposition $(S, g)$ of $Y$ with connected binding $K \subset Y^{1}$. If the genus of $S$ is $k$ then the knot Floer homology, $\widehat{H F K}(-Y, K,-k) \cong \mathbb{Z}$. Moreover, it is possible to find a Heegaard diagram for which the knot Floer homology in this $-k$ th filtration level is generated by an element of the chain complex $\widehat{C F K}(-Y, K)$ which also represents a cycle in the chain complex $\widehat{C F}(-Y)$. The contact invariant $c(\xi)$ is defined to be the image of this cycle in $\widehat{H F}(-Y)$.

The class $c(\xi)$ is well-defined up to sign (we use $\mathbb{Z}_{2}$ coefficients throughout to avoid ambiguity in sign), and it is an invariant of $\xi$ up to isotopy of the contact structure. This invariant encodes information related to the tightness of $\xi$. For instance, Ozsváth and Szabó prove that if $\xi$ is overtwisted, then $c(\xi)=0$. On the other hand, if $\xi$ is Stein fillable or strongly fillable, then $c(\xi) \neq 0$ [12], [11]. In a previous paper we show, in the case of contact structures compatible with genus one, one boundary component open books, that $c(\xi)=0$ if and only if $\xi$ is overtwisted for all but a small family of open books with reducible monodromies [1]. Honda, Kazez, and Matić have since shown this to be the case for all such open books [7], [9], [8]. However, the precise relationship between $c(\xi)$ and the tightness of $\xi$ is still unknown - there are tight contact structures with vanishing contact invariant [4]. In fact, Ghiggini, Honda, and Van Horn-Morris recently showed that the contact invariant vanishes for contact structures with positive Giroux torsion [5].

\footnotetext{
Received by the editors March 13, 2007.

${ }^{1}$ Here, $S$ is a surface with boundary, and $g$ is a diffeomorphism of $S$ restricting to the identity of $\partial S$
} 
As the contact invariant is defined in terms of a compatible open book decomposition, we often denote $c(\xi)$ by $c(S, g)$. This class satisfies the following naturality property [12]:

Theorem 1.1 (Ozsváth-Szabó). If $(S, g)$ is an open book decomposition for $Y$, and $\gamma \subset Y-L$ is a curve supported in a page of the open book ( $L$ is the binding), which is not homotopic to the boundary, then $\left(S, t_{\gamma}^{-1} \mathrm{~g}\right)$ induces an open book decomposition of $Y_{+1}(\gamma)$ (here, $t_{\gamma}$ denotes the right-handed Dehn twist around $\gamma$ ). And under the map

$$
F_{W}: \widehat{H F}(-Y) \longrightarrow \widehat{H F}\left(-Y_{+1}(\gamma)\right)
$$

obtained by the two-handle addition (and summing over all spin ${ }^{c}$ structures), we have that

$$
F_{W}(c(S, g))= \pm c\left(S, t_{\gamma}^{-1} g\right)
$$

In general, it is an open question as to whether tightness of the contact structures compatible with open books $(S, g)$ and $(S, h)$ implies tightness of the contact structure compatible with $(S, h g)$. This question is open even when $h$ is a right-handed Dehn twist $t_{\gamma}$ around a curve $\gamma \subset S[3]$. Along these lines, however, Theorem 1.1 implies that if $c(S, g) \neq 0$, then $c\left(S, t_{\gamma} \circ g\right) \neq 0$. This behavior is generalized in Theorem 1.2, which follows from our main result.

Theorem 1.2. Suppose that $g$ and $h$ are diffeomorphisms of $S$ which restrict to the identity on $\partial S$ and that $c(S, g) \neq 0$ and $c(S, h) \neq 0$. Then $c(S, h g) \neq 0$.

This theorem has the immediate corollary:

Corollary 1.3. If the contact structures compatible with $(S, g)$ and $(S, h)$ are strongly fillable, then the contact structure compatible with $(S, h g)$ is tight.

Another formulation of Theorem 1.2 is the statement that for a fixed $S$ the set

$$
N_{S}=\{g \in \operatorname{Aut}(S, \partial S) \mid c(S, g) \neq 0\}
$$

is a monoid under composition of diffeomorphisms, where here $\operatorname{Aut}(S, \partial S)$ denotes the set of isotopy classes of diffeomorphisms of $S$ which restrict to the identity on $\partial S$. As mentioned above, it is unknown whether the set of diffeomorphisms of $S$ giving tight contact structures forms a monoid under composition. On the other hand, there are a few notable monoids of this flavor. Two rather obvious ones are the set of diffeomorphisms of $S$ expressible as products of positive Dehn twists ${ }^{2}$, and the set of right-veering diffeomorphisms of $S$, which feature prominently in the classification results of Honda, Kazez, and Matić mentioned earlier.

The key to Theorem 1.2 is the observation that the contact invariant satisfies the naturality property described below. For any $g$ and $h$ we exhibit a cobordism $X_{g, h}$ with

$$
\partial X_{g, h}=-Y_{g}-Y_{h}+Y_{h g}
$$

\footnotetext{
${ }^{2}$ Note that this is not necessarily equivalent to the set of diffeomorphisms giving Stein fillable contact structures. In fact, it is presently unknown (though thought to be the case) whether the Stein fillability of $(S, g)$ and $(S, h)$ implies Stein fillability of $(S, h g)$.
} 
where $Y_{\phi}$ is the 3-manifold with open book decomposition $(S, \phi)$. This cobordism induces a chain map (multiplication)

$$
m: \widehat{C F}\left(Y_{g}\right) \otimes_{\mathbb{Z}_{2}} \widehat{C F}\left(Y_{h}\right) \rightarrow \widehat{C F}\left(Y_{h g}\right) .
$$

If we apply the $\operatorname{Hom}_{\mathbb{Z}_{2}}\left(-, \mathbb{Z}_{2}\right)$ functor to this expression, we obtain a chain map (comultiplication)

$$
\mu: \widehat{C F}\left(-Y_{h g}\right) \rightarrow \widehat{C F}\left(-Y_{g}\right) \otimes_{\mathbb{Z}_{2}} \widehat{C F}\left(-Y_{h}\right) .
$$

We show that the contact invariants are natural under the corresponding map

$$
\tilde{\mu}: \widehat{H F}\left(-Y_{h g}\right) \rightarrow \widehat{H F}\left(-Y_{g}\right) \otimes_{\mathbb{Z}_{2}} \widehat{H F}\left(-Y_{h}\right)
$$

induced on homology. That is

Theorem 1.4. The map $\tilde{\mu}$ takes $c(S, h g) \mapsto c(S, g) \otimes c(S, h)$.

Hence, if $c(S, h g)=0$ then either $c(S, g)=0$ or $c(S, h)=0$, and Theorem 1.2 follows immediately.

In Section 4, we generalize the result of Theorem 1.4 by examining analogous maps on $\mathrm{HF}^{+}$. We use this generalization to prove the following theorem.

Theorem 1.5. If $c^{+}(S, h g) \in U^{d} \cdot H F^{+}\left(-Y_{h g}\right)$, then $c^{+}\left(S \#_{b} S, g \# h\right) \in$ $U^{d} \cdot H F^{+}\left(-\left(Y_{g} \# Y_{h}\right)\right)$.

In Theorem 1.5, $c^{+}(S, \phi)$ denotes the image in $H F^{+}\left(-Y_{\phi}\right)$ of $c(S, \phi)$ under the natural map $\widehat{H F}\left(-Y_{\phi}\right) \rightarrow H F^{+}\left(-Y_{\phi}\right)$. Furthermore, if $(S, g)$ and $(S, h)$ are open books compatible with contact structures $\left(Y_{g}, \xi_{g}\right)$ and $\left(Y_{h}, \xi_{h}\right)$, respectively, then $\left(S \#_{b} S, g \# h\right)$ is an open book compatible with the contact structure $\left(Y_{g} \# Y_{h}, \xi_{g} \# \xi_{h}\right)$, where $\#_{b}$ denotes boundary connected sum.

The following corollaries of Theorem 1.5 provide obstructions to a contact 3manifold with open book $(S, h g)$ having a compatible open book with planar pages. At the same time, we obtain obstructions to the 3-manifold $Y_{h g}$ being an $L$-space. The reader should compare these corollaries with those found by Ozsváth, Szabó, and Stipsicz in [13]. In what follows, $\mathfrak{s}(S, \phi)$ denotes the spin ${ }^{c}$ structure associated to the contact 2-plane field which is compatible with $(S, \phi)$.

Corollary 1.6. Suppose that $c^{+}\left(S \#{ }_{b} S, g \# h\right) \neq 0$ and that $c_{1}(\mathfrak{s}(S, g))$ is non-torsion. Then $Y_{h g}$ is not an L-space and the contact structure corresponding to $(S, h g)$ is not compatible with a planar open book.

Corollary 1.7. Suppose that $(S, g)$ and $(S, h)$ correspond to Stein fillable contact manifolds $\left(Y_{g}, \xi_{g}\right)$ and $\left(Y_{h}, \xi_{h}\right)$ with fillings $\left(X_{g}, J_{g}\right)$ and $\left(X_{h}, J_{h}\right)$. Suppose further that $c_{1}\left(\mathfrak{s}\left(\xi_{g}\right)\right)=0=c_{1}\left(\mathfrak{s}\left(\xi_{h}\right)\right)$ and $c_{1}\left(X_{g}, J_{g}\right) \neq 0$. Then $Y_{h g}$ is not an L-space and the contact structure corresponding to $(S, h g)$ is not compatible with a planar open book.

\section{Heegaard diagrams and the contact class}

Honda, Kazez, and Matić give another interpretation of the Ozsváth-Szabó contact class in [7]. We use their reformulation in our proof of Theorem 1.2. Recall that the 
open book $(S, g)$ is a decomposition of the 3-manifold $Y=S \times[0,1] / \sim$, where $\sim$ is the relation defined by

$$
\begin{aligned}
(x, 1) \sim(g(x), 0), & x \in S \\
(x, t) \sim(x, s), & x \in \partial S, t, s \in[0,1] .
\end{aligned}
$$

$Y$ has a Heegaard splitting $Y=H_{1} \cup H_{2}$, where $H_{1}$ is the handlebody $S \times[0,1 / 2]$ and $H_{2}$ is the handlebody $S \times[1 / 2,1]$. Let $S_{t}$ denote the page $S \times\{t\}$. If $S$ has $n$ boundary components and genus $k$ then the Heegaard surface in this splitting is the genus $2 k+n-1$ surface $\Sigma=S_{1 / 2} \cup-S_{0}$. To specify a pointed Heegaard diagram for $Y$ it remains to describe the $\alpha$ and $\beta$ curves on $\Sigma$ and the placement of a basepoint $z$. Choose $2 k+n-1$ disjoint properly embedded arcs $a_{1}, \ldots, a_{2 k+n-1}$ on $S$ so that $S \backslash \cup a_{i}$ is topologically a disk. For each $i$ we obtain $b_{i}$ by changing the $\operatorname{arcs} a_{i}$ via a small isotopy which moves the endpoints of the $a_{i}$ along $\partial S$ in the direction given by the orientation of $\partial S$ so that $a_{i}$ intersects $b_{i}$ transversely in one point and with positive $\operatorname{sign}\left(\right.$ where $b_{i}$ inherits its orientation from $a_{i}$ ). See Figure 1 for an illustration of the $a_{i}$ and $b_{i}$ arcs on a surface $S$.

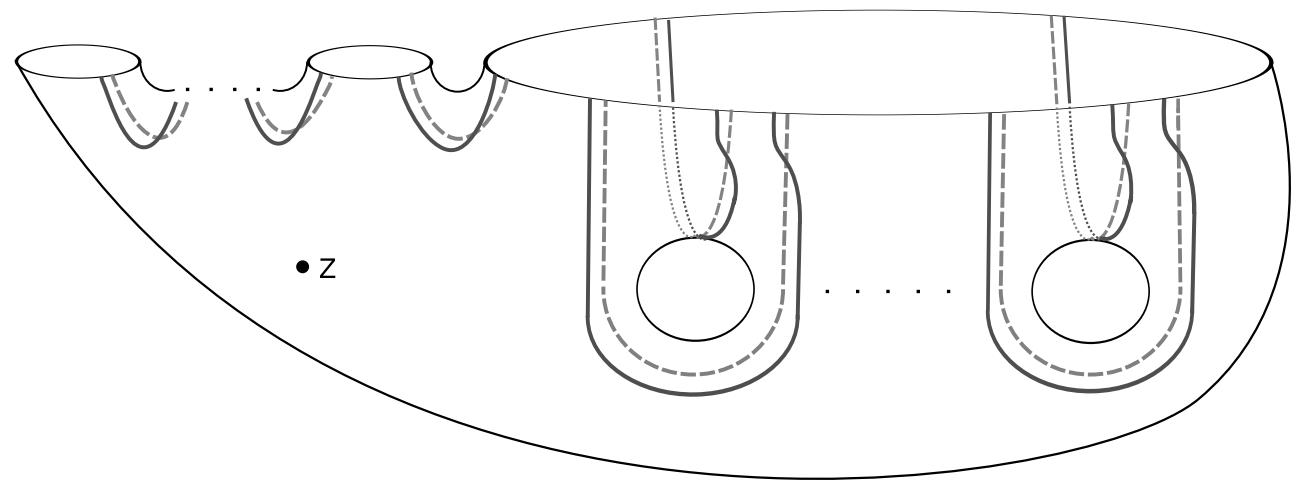

Figure 1. A surface $S$ with multiple boundary components and genus greater than one. The $a_{i}$ arcs are dashed and the $b_{i}$ arcs are solid.

Now define

$$
\begin{gathered}
\alpha_{i}=a_{i} \times\{1 / 2\} \cup a_{i} \times\{0\} \\
\beta_{i}=b_{i} \times\{1 / 2\} \cup g\left(b_{i}\right) \times\{0\} .
\end{gathered}
$$

Place the basepoint $z$ in the big region on $S_{1 / 2}$ (that is, not in one of the thin strip regions). For each $i=1, \ldots, 2 k+n-1$ let $x_{i}$ be the intersection point on $S_{1 / 2}$ between $\alpha_{i}$ and $\beta_{i}$. Then $\overline{x_{g}}=\left\{x_{1}, \ldots, x_{2 k+n-1}\right\}$ is an intersection point between $\mathbb{T}_{\alpha}$ and $\mathbb{T}_{\beta}$ in $S y m^{2 k+n-1}(\Sigma)$. Moreover, $\overline{x_{g}}$ is a cycle in $\operatorname{Hom}\left(\widehat{C F}(Y), \mathbb{Z}_{2}\right)=\widehat{C F}(-Y)$ because of the placement of $z$. See Figure 2 for an illustration of the pointed Heegaard diagram for an open book.

Theorem 2.1 (Honda-Kazez-Matić). $\left[\overline{x_{g}}\right] \in \widehat{H F}(-Y)$ is the Ozsváth-Szabó contact class $c(S, g)$. 

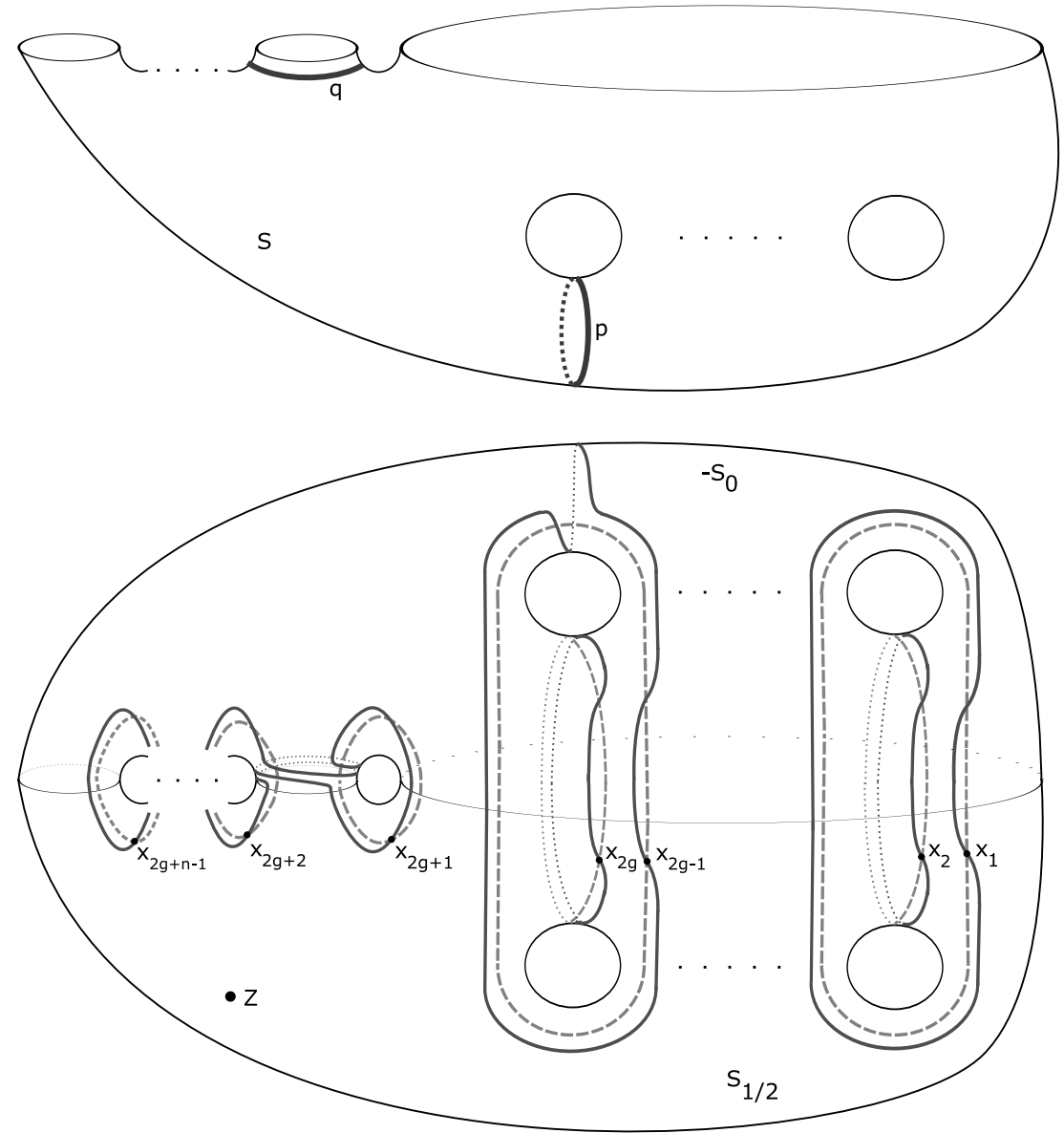

FIguRE 2. The bottom figure is a pointed Heegaard diagram for the open book $(S, g)$, where $S$ is a genus $k$ surface with $n$ boundary components and $g$ is the composition of a left-handed Dehn twist around the curve $p$ with a right-handed Dehn twist around the curve $q$. The $\alpha_{i}$ are dashed and the $\beta_{i}$ are solid. We have also labeled the intersection point $\overline{x_{g}}=\left\{x_{1}, \ldots, x_{2 k+n-1}\right\}$.

\section{Naturality under comultiplication}

Given a surface $S$ with genus $k$ and $n$ boundary components, let $a_{i}$ and $b_{i}$ be the set of properly embedded arcs described above. We construct another set of disjoint properly embedded $\operatorname{arcs} c_{i}$ from the $b_{i}$ by changing the $\operatorname{arcs} b_{i}$ via a small isotopy which moves the endpoints of the $b_{i}$ along $\partial S$ in the direction given by the orientation of $\partial S$. We require that both $a_{i}$ and $b_{i}$ intersect $c_{i}$ transversely in one point and with positive sign (where $c_{i}$ inherits its orientation from $b_{i}$ ). For any two diffeomorphisms $g$ and $h$, we construct three sets of attaching curves on the Heegaard surface $\Sigma=S_{1 / 2} \cup-S_{0}$ :

$$
\alpha_{i}=a_{i} \times\{1 / 2\} \cup a_{i} \times\{0\}
$$




$$
\begin{gathered}
\beta_{i}=b_{i} \times\{1 / 2\} \cup g\left(b_{i}\right) \times\{0\} \\
\gamma_{i}=c_{i} \times\{1 / 2\} \cup h\left(g\left(c_{i}\right)\right) \times\{0\} .
\end{gathered}
$$

Once again, we place the basepoint $z$ in the big region of $S_{1 / 2}$ (outside of the thin strip regions). Then $(\Sigma, \bar{\alpha}, \bar{\beta}, \bar{\gamma}, z)$ is a pointed Heegaard triple-diagram and can be used as in [11] to construct a cobordism $X_{\alpha, \beta, \gamma}$ with

$$
\partial X_{\alpha, \beta, \gamma}=-Y_{\alpha, \beta}-Y_{\beta, \gamma}+Y_{\alpha, \gamma}
$$

where $Y_{\alpha, \beta}$ is the three manifold with Heegaard decomposition $(\Sigma, \bar{\alpha}, \bar{\beta}$ ) (and similarly for $Y_{\beta, \gamma}$ and $\left.Y_{\alpha, \gamma}\right)$. Such a cobordism induces a chain map

$$
\widehat{C F}\left(Y_{\alpha, \beta}\right) \otimes_{\mathbb{Z}_{2}} \widehat{C F}\left(Y_{\beta, \gamma}\right) \rightarrow \widehat{C F}\left(Y_{\alpha, \gamma}\right) .
$$

By the description of the Heegaard diagram associated to an open book in section 2, it is clear that

$$
\begin{gathered}
Y_{\alpha, \beta}=Y_{g} \\
Y_{\beta, \gamma}=Y_{h} \\
Y_{\alpha, \gamma}=Y_{h g} .
\end{gathered}
$$

Thus, we have a chain map

$$
m: \widehat{C F}\left(Y_{g}\right) \otimes_{\mathbb{Z}_{2}} \widehat{C F}\left(Y_{h}\right) \rightarrow \widehat{C F}\left(Y_{h g}\right) .
$$

If the pointed Heegaard triple-diagram $(\Sigma, \bar{\alpha}, \bar{\beta}, \bar{\gamma}, z)$ is weakly-admissible then this map is defined on the generators of $\widehat{C F}\left(Y_{g}\right) \otimes_{\mathbb{Z}_{2}} \widehat{C F}\left(Y_{h}\right)$ by

$$
m(\bar{a} \otimes \bar{b})=\sum_{\bar{x} \in \mathbb{T}_{\alpha} \cap \mathbb{T}_{\gamma}} \sum_{\left\{\phi \in \pi_{2}(\bar{a}, \bar{b}, \bar{x})\right.}(\# \mathcal{\lambda}(\phi)) \bar{x} .
$$

In this sum, $\pi_{2}(\bar{a}, \bar{b}, \bar{x})$ is the set of homotopy classes of Whitney triangles connecting $\bar{a}, \bar{b}$, and $\bar{x} ; \lambda(\phi)$ is the expected dimension of the moduli space of holomorphic representatives of $\phi ; n_{z}(\phi)$ is the algebraic intersection number of $\phi$ with the subvariety $\{z\} \times \operatorname{Sym}^{2 k+n-2}(\Sigma) \subset S y m^{2 k+n-1}(\Sigma)$; and $\mathcal{M}(\phi)$ is the moduli space of holomorphic representatives of $\phi$. For more details, see [11].

As alluded to in the introduction, we apply the $\operatorname{Hom}_{\mathbb{Z}_{2}}\left(-, \mathbb{Z}_{2}\right)$ functor to the expression in equation 1 . If we represent each chain complex diagrammatically by drawing an arrow from $x$ to $y$ whenever $y$ is a term in $\partial x$ or when $y$ is a term in the image of $x$ under the map $m$, then applying the $\operatorname{Hom}_{\mathbb{Z}_{2}}\left(-, \mathbb{Z}_{2}\right)$ functor corresponds to reversing the direction of every arrow. Doing so, we obtain a chain map

$$
\mu: \widehat{C F}\left(-Y_{h g}\right) \rightarrow \widehat{C F}\left(-Y_{g}\right) \otimes_{\mathbb{Z}_{2}} \widehat{C F}\left(-Y_{h}\right) .
$$

An element in $\widehat{C F}\left(-Y_{h g}\right)$ is a sum of intersection points $\bar{x} \in \mathbb{T}_{\alpha} \cap \mathbb{T}_{\gamma}$. On such an intersection point the chain map $\mu$ is defined by

$$
\mu(\bar{x})=\sum_{\bar{a} \in \mathbb{T}_{\alpha} \cap \mathbb{T}_{\beta}, \bar{b} \in \mathbb{T}_{\beta} \cap \mathbb{T}_{\gamma}} \sum_{\left\{\phi \in \pi_{2}(\bar{a}, \bar{b}, \bar{x})\right.}(\# \mathcal{M}(\phi))(\bar{a} \otimes \bar{b})
$$

as long as the pointed Heegaard triple-diagram $(\Sigma, \bar{\alpha}, \bar{\beta}, \bar{\gamma}, z)$ is weakly-admissible. To prove Theorem 1.4, we show that

$$
\mu\left(\overline{x_{h g}}\right)=\overline{x_{g}} \otimes \overline{x_{h}} .
$$


We complete the proof in two steps. First we show that $(\Sigma, \bar{\alpha}, \bar{\beta}, \bar{\gamma}, z)$ is weaklyadmissible. Then we show that there is only one pair $\left\{\bar{a} \in \mathbb{T}_{\alpha, \beta}, \bar{b} \in \mathbb{T}_{\beta, \gamma}\right\}$ for which there exists a homotopy class $\phi \in \pi_{2}\left(\bar{a}, \bar{b}, \overline{x_{h g}}\right)$ with $n_{z}(\phi)=0$ and such that $\phi$ has a holomorphic representative. Moreover, $\bar{a}=\overline{x_{g}}, \bar{b}=\overline{x_{h}}$, and the number of holomorphic representatives of $\phi$ is one.

3.1. Weak Admissibility. We begin with two definitions from [11].

Definition 3.1. For a pointed Heegaard triple-diagram $(\Sigma, \bar{\alpha}, \bar{\beta}, \bar{\gamma}, z)$, let $D_{1}, \ldots, D_{m}$ be the connected regions of $\Sigma-\cup \alpha_{i}-\cup \beta_{i}-\cup \alpha_{i}$. And let $\psi=\sum_{i} p_{i} D_{i}$ be a formal linear combination of the $D_{i}$ so that $n_{z}(\psi)=0$ and $\partial \psi=\sum_{i} p_{i} \partial D_{i}$ is a linear combination of complete $\alpha, \beta$, and $\gamma$ curves. Then $\psi$ is called a triply-periodic domain.

Definition 3.2. The pointed Heegaard triple-diagram is said to be weakly-admissible if every non-trivial triply-periodic domain $\psi$ has both positive and negative coefficients.

For each $i=1, \ldots, 2 k+n-1$ the curves $\alpha_{i}, \beta_{i}$, and $\gamma_{i}$ intersect on $S_{1 / 2}$ in the arrangement depicted in Figure 3.

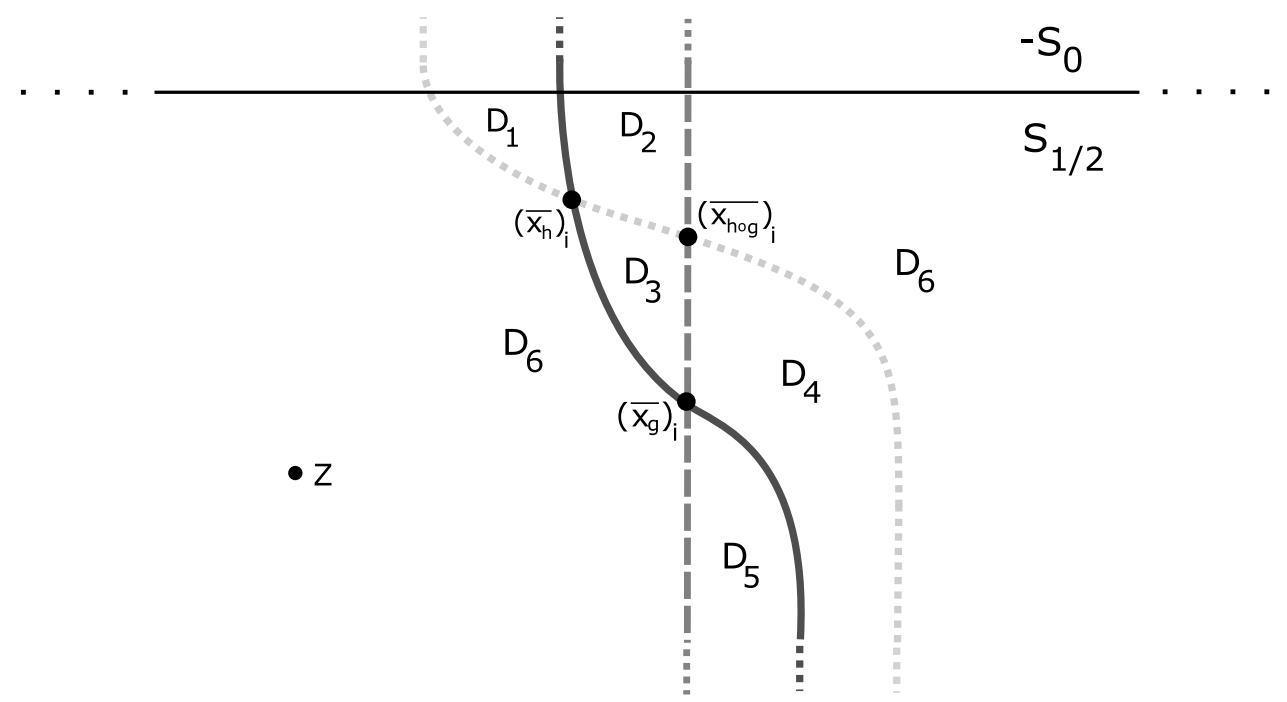

Figure 3. The curve $\alpha_{i}$ is dashed, $\beta_{i}$ is solid, and $\gamma_{i}$ is dotted. We have labeled regions $D_{1}, \ldots, D_{6}$ as well as the $i t h$ components of the various contact classes $\overline{x_{g}}, \overline{x_{h}}$, and $\overline{x_{h g}}$.

If $\psi=\sum_{j} p_{j} D_{j}$ is a triply-periodic domain, then $p_{6}=0$ since $D_{6}$ contains the basepoint $z$. Since $\partial \psi$ includes some number of complete $\alpha_{i}$ curves,

$$
p_{2}=p_{3}-p_{4}=-p_{5} \text {. }
$$

Therefore, $\psi$ has both positive and negative coefficients unless $p_{2}=p_{5}=0$ and $p_{3}=p_{4}$. So let's assume the latter. Since $\partial \psi$ includes some number of complete $\beta_{i}$ curves,

$$
p_{1}=-p_{3}=0
$$


So, either $\psi$ has both positive and negative coefficients or $p_{1}=\cdots=p_{6}=0$ and $\partial \psi$ includes no $\alpha_{i}, \beta_{i}$, or $\gamma_{i}$ curves. If we carry out this analysis for all $i=1, \ldots, 2 k+n-1$ we see that either $\psi$ has both positive and negative coefficients or else it is trivial. Hence $(\Sigma, \bar{\alpha}, \bar{\beta}, \bar{\gamma}, z)$ is weakly-admissible.

3.2. Completing the proof of Theorem 1.4. Let $\Delta$ denote the 2 -simplex and label its vertices clockwise $v_{\alpha}, v_{\beta}, v_{\gamma}$. Let $e_{\alpha}$ be the edge opposite $v_{\alpha}$ (and similarly for $e_{\beta}$ and $e_{\gamma}$ ). The boundary of $\Delta$ inherits the standard counterclockwise orientation. Recall the following definition from [11]:

Definition 3.3. A map $u: \Delta \rightarrow \operatorname{Sym}^{2 k+n-1}(\Sigma)$ satisfying $u\left(v_{\gamma}\right)=\bar{a}, u\left(v_{\alpha}\right)=\bar{b}$, and $u\left(v_{\beta}\right)=\overline{x_{h g}}$, and $u\left(e_{\alpha}\right) \subset \mathbb{T}_{\alpha}, u\left(e_{\beta}\right) \subset \mathbb{T}_{\beta}$, and $u\left(e_{\gamma}\right) \subset \mathbb{T}_{\gamma}$ is called a Whitney triangle between $\bar{a}, \bar{b}$, and $\overline{x_{h g}}$. This map $u$ is represented schematically in Figure 4.

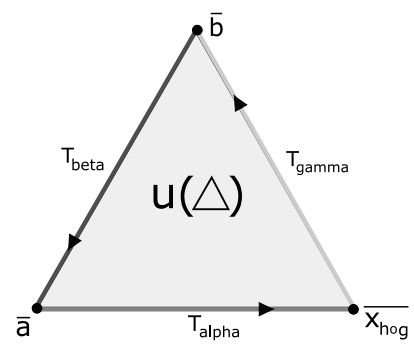

Figure 4.

We can represent $\phi \in \pi_{2}\left(\bar{a}, \bar{b}, \overline{x_{h g}}\right)$ by a 2 -chain $\widehat{\phi}=\sum_{j} p_{j} D_{j}$ whose oriented boundary consists of $\alpha$ arcs from $\bar{a}$ to $\overline{x_{h g}}, \beta$ arcs from $\bar{b}$ to $\bar{a}$, and $\gamma$ arcs from $\overline{x_{h g}}$ to $\bar{b}$. Suppose $n_{z}(\phi)=0$ and $\phi$ has a holomorphic representative. Then $n_{z}(\widehat{\phi})=0$ and the $p_{j}$ are all non-negative. We refer to Figure 3 for the local picture near the ith component of the contact classes $\overline{x_{g}}, \overline{x_{h}}$, and $\overline{x_{h g}}$. Write

$$
\widehat{\phi}=p_{1} D_{1}+\cdots+p_{6} D_{6}+\sum_{j>6} p_{j} D_{j} .
$$

Now we can analyze the possibilities for $p_{1}, \ldots, p_{6}$ given the boundary constraints on $\widehat{\phi} \cdot\left(\overline{x_{h g}}\right)_{i}$ must be a corner of the region defined by $\widehat{\phi}$; moreover this corner is such that we enter $\left(\overline{x_{h g}}\right)_{i}$ along an arc of $\alpha_{i}$ and we leave along an arc of $\gamma_{i}$. Therefore, $p_{6}+p_{3}=p_{2}+p_{4}+1$. If $\left(\overline{x_{h}}\right)_{i}$ is not a corner, then $p_{3}+p_{1}=p_{2}+p_{6}$. Note that $p_{6}=0$ since $n_{z}(\widehat{\phi})=0$. Thus, these two equations become

$$
\begin{aligned}
p_{3} & =p_{2}+p_{4}+1 \\
p_{3}+p_{1} & =p_{2} .
\end{aligned}
$$

Subtracting the second equation from the first, we have

$$
-p_{1}=p_{4}+1
$$

which implies that either $p_{1}$ or $p_{4}$ is negative, which cannot happen since $\phi$ has a holomorphic representative. Therefore, $\left(\overline{x_{h}}\right)_{i}$ is a corner. The same type of analysis shows that $\left(\overline{x_{g}}\right)_{i}$ is a corner. 
Since $\left(\overline{x_{h}}\right)_{i}$ is a corner, either $p_{1}+p_{3}+1=p_{2}$ or $p_{1}+p_{3}=p_{2}+1$. Substituting $p_{3}=p_{2}+p_{4}+1$ into both expressions, we have the two possibilities $p_{1}+p_{2}+p_{4}+2=p_{2}$ or $p_{1}+p_{2}+p_{4}=p_{2}$. We can rule out the first possibility as it implies that either $p_{1}$ or $p_{4}$ is negative. And the second possibility holds only if $p_{1}=p_{4}=0$. So, to summarize what we know so far: $p_{1}=0, p_{3}=p_{2}+1, p_{4}=0$, and $p_{6}=0$.

Since $\left(\overline{x_{g}}\right)_{i}$ is a corner, then either $p_{5}+p_{3}=p_{4}+p_{6}+1$ or $p_{5}+p_{3}+1=p_{4}+p_{6}$. Substituting what we know of $p_{3}, p_{4}$, and $p_{6}$ into these two expressions, we obtain the two possibilities $p_{5}+p_{2}+1=1$ or $p_{5}+p_{2}+2=0$. We can rule out the second possibility as it implies that either $p_{5}$ or $p_{2}$ is negative. And the first possibility holds only if $p_{5}=p_{2}=0$. Thus, we have determined that the only possibility for the values $p_{1}, \ldots, p_{6}$ are:

$$
\begin{aligned}
p_{1}=p_{2}= & p_{4}=p_{5}=p_{6}=0 \\
p_{3} & =1 .
\end{aligned}
$$

Because the same analysis works for every $i=1, \ldots, 2 k+n-1$ and because every component of $\partial \widehat{\phi}$ must contain some $\left(\overline{x_{h g}}\right)_{i}$ we can conclude that $\widehat{\phi}$ is the linear combination which is the sum of precisely one of these small triangular regions $\left(D_{3}\right.$ in figure 3) for each $i$. Therefore, any holomorphic triangle $\phi$ between $\bar{a}, \bar{b}$, and $\overline{x_{h g}}$ with $n_{z}(\phi)=0$ is, in fact, a triangle between $\overline{x_{g}}, \overline{x_{h}}$, and $\overline{x_{h g}}$, and can be expressed as a product of these small triangles in our Heegaard diagram. Moreover, since each of these disjoint triangular regions is topologically a disk, and we have specified the image of three boundary points, $\# \mathcal{M}(\phi)=1$ by the Riemann Mapping Theorem. Hence,

$$
\mu\left(\overline{x_{h g}}\right)=\overline{x_{g}} \otimes \overline{x_{h}} .
$$

Therefore,

$$
\tilde{\mu}(c(S, h g))=c(S, g) \otimes c(S, h)
$$

and the proof of Theorem 1.4 is complete. As mentioned in the Introduction, Theorem 1.2 follows immediately.

\section{A generalization of Theorem 1.4}

4.1. $C F^{\leq 0}$ and connected sums. For a $\operatorname{spin}^{c}$ structure $\mathfrak{s}$ on $Y$ and a pointed Heegaard diagram $(\Sigma, \bar{\alpha}, \bar{\beta}, z)$ for $Y$ which is strongly $\mathfrak{s}$-admissible, recall that we can define a chain complex $C F^{\leq 0}(Y, \mathfrak{s})$ which is finitely generated as a $\mathbb{Z}_{2}[U]$ module [11]. The generators of $C F^{\leq 0}(Y, \mathfrak{s})$ are pairs of the form $[\bar{x}, i]$, where $\bar{x} \in \mathbb{T}_{\alpha} \cap \mathbb{T}_{\beta}, \mathfrak{s}_{z}(\bar{x})=\mathfrak{s}$, $i \in \mathbb{Z}^{\leq 0}$, and $U$ acts by $U \cdot[\bar{x}, i]=[\bar{x}, i-1]$. The differential on $C F^{\leq 0}(Y, \mathfrak{s})$ is given by

$$
\partial[\bar{x}, i]=\sum_{\bar{y} \in \mathbb{T}_{\alpha} \cap \mathbb{T}_{\gamma}} \sum_{\left\{\phi \in \pi_{2}(\bar{x}, \bar{y}) \mid \lambda(\phi)=1\right\}} \#\left(\frac{\mathcal{M}(\phi)}{\mathbb{R}}\right) \cdot\left[\bar{y}, i-n_{z}(\phi)\right] .
$$

We can identify $\widehat{C F}(Y, \mathfrak{s})$ with $C F^{\leq 0}(Y, \mathfrak{s}) / U \cdot C F^{\leq 0}(Y, \mathfrak{s})$, so there is a natural quotient map

$$
\pi: C F^{\leq 0}(Y, \mathfrak{s}) \rightarrow \widehat{C F}(Y, \mathfrak{s}) .
$$

If $A$ is a $\mathbb{Z}_{2}[U]$ module, let $A^{\vee}$ denote $\operatorname{Hom}_{\mathbb{Z}_{2}[U]}\left(A, \mathbb{Z}_{2}\left[U, U^{-1}\right] / \mathbb{Z}_{2}[U]\right)$. Then observe that we can identify $C F^{\leq 0}(Y, \mathfrak{s})^{\vee}$ with $C F^{+}(-Y, \mathfrak{s})$, and $\widehat{C F}(Y, \mathfrak{s})^{\vee}$ with $\widehat{C F}(-Y, \mathfrak{s})$ 
as complexes over $\mathbb{Z}_{2}[U]$ and $\mathbb{Z}_{2}$, respectively. ${ }^{3}$ In fact, applying the $\operatorname{Hom}_{\mathbb{Z}_{2}[U]}\left(-, \mathbb{Z}_{2}\left[U, U^{-1}\right] / \mathbb{Z}_{2}[U]\right)$ functor to the expression above, we obtain the natural inclusion map

$$
\pi^{\vee}: \widehat{C F}(-Y, \mathfrak{s}) \rightarrow C F^{+}(-Y, \mathfrak{s})
$$

which sends

$$
\bar{x} \mapsto[\bar{x}, 0]
$$

for $\bar{x} \in \mathbb{T}_{\alpha} \cap \mathbb{T}_{\beta}$.

In [10] the authors construct a homotopy equivalence

$$
f^{\leq 0}: C F^{\leq 0}\left(Y_{1}, \mathfrak{s}_{1}\right) \otimes_{\mathbb{Z}_{2}[U]} C F^{\leq 0}\left(Y_{2}, \mathfrak{s}_{2}\right) \rightarrow C F^{\leq 0}\left(Y_{1} \# Y_{2}, \mathfrak{s}_{1} \# \mathfrak{s}_{2}\right)
$$

as follows. Let $\left(\Sigma_{i}, \bar{\alpha}_{i}, \bar{\beta}_{i}, z_{i}\right)$ be a strongly $\mathfrak{s}_{i}$-admissible pointed Heegaard diagram for $Y_{i}$, for $i=1,2$. Consider the pointed Heegaard triple-diagram $\left(\Sigma_{1} \# \Sigma_{2}, \bar{\alpha}_{1} \cup\right.$ $\left.\bar{\alpha}_{2}^{\prime}, \bar{\beta}_{1} \cup \bar{\alpha}_{2}, \bar{\beta}_{1}^{\prime} \cup \bar{\beta}_{2}, z\right)$, where the connected sum of $\Sigma_{1}$ with $\Sigma_{2}$ occurs at the $z_{i}, z$ is a point in the connected sum region, and $\bar{\alpha}_{2}^{\prime}$ and $\bar{\beta}_{1}^{\prime}$ are exact Hamiltonian translates of $\bar{\alpha}_{2}$ and $\bar{\beta}_{1}$ so that this new diagram is admissible. Suppose that the genera of $\Sigma_{1}$ and $\Sigma_{2}$ are $g_{1}$ and $g_{2}$ and let $\Theta_{1} \in \mathbb{T}_{\beta_{1}} \cap \mathbb{T}_{\beta_{1}^{\prime}}$ and $\Theta_{2} \in \mathbb{T}_{\alpha_{2}} \cap \mathbb{T}_{\alpha_{2}^{\prime}}$ be the top graded intersection points in $\widehat{C F}\left(\#^{g_{1}}\left(S^{1} \times S^{2}\right), \mathfrak{s}_{0}\right)$ and $\widehat{C F}\left(\#^{g_{2}}\left(S^{1} \times S^{2}\right), \mathfrak{s}_{0}\right)$. Then the maps $[\bar{x}, i] \mapsto\left[\bar{x} \times \Theta_{2}, i\right]$ and $[\bar{y}, j] \mapsto\left[\Theta_{1} \times \bar{y}, j\right]$ define chain maps

$$
\Phi_{1}: C F^{\leq 0}\left(Y_{1}, \mathfrak{s}_{1}\right) \rightarrow C F^{\leq 0}\left(Y_{1} \# \#^{g_{2}}\left(S^{1} \times S^{2}\right), \mathfrak{s}_{1} \# \mathfrak{s}_{0}\right),
$$

and

$$
\Phi_{2}: C F^{\leq 0}\left(Y_{2}, \mathfrak{s}_{2}\right) \rightarrow C F^{\leq 0}\left(\#^{g_{2}}\left(S^{1} \times S^{2}\right) \# Y_{2}, \mathfrak{s}_{0} \# \mathfrak{s}_{2}\right) .
$$

The pointed Heegaard triple-diagram above can be used to define a map

$$
\begin{aligned}
\Gamma: C F^{\leq 0}\left(Y_{1} \# \#^{g_{2}}\left(S^{1} \times S^{2}\right), \mathfrak{s}_{1} \# \mathfrak{s}_{0}\right) \otimes_{\mathbb{Z}_{2}[U]} C F^{\leq 0}\left(\#^{g_{2}}\left(S^{1} \times S^{2}\right) \# Y_{2}, \mathfrak{s}_{0} \# \mathfrak{s}_{2}\right) \\
\longrightarrow C F^{\leq 0}\left(Y_{1} \# Y_{2}, \mathfrak{s}_{1} \# \mathfrak{s}_{2}\right) .
\end{aligned}
$$

The map $f^{\leq 0}$ is then defined to be

$$
f^{\leq 0}=\Gamma \circ\left(\Phi_{1} \otimes \Phi_{2}\right) .
$$

The maps in this composition are all $U$-equivariant by construction; hence, so is $f \leq 0$. In [10], Oszváth and Szabó show that $f \leq 0$ is a homotopy equivalence. We define a homotopy equivalence

$$
f: \widehat{C F}\left(Y_{1}, \mathfrak{s}_{1}\right) \otimes_{\mathbb{Z}_{2}} \widehat{C F}\left(Y_{2}, \mathfrak{s}_{2}\right) \rightarrow \widehat{C F}\left(Y_{1} \# Y_{2}, \mathfrak{s}_{1} \# \mathfrak{s}_{2}\right)
$$

in exactly the same way. ${ }^{4}$

\footnotetext{
${ }^{3}$ We have already been identifying $\widehat{C F}(-Y)$ with $\operatorname{Hom}_{\mathbb{Z}_{2}}\left(\widehat{C F}(Y), \mathbb{Z}_{2}\right)$, but the latter is isomorphic as a $\mathbb{Z}_{2}$ module to $\widehat{C F}(Y)^{\vee}$ since we are thinking of $\widehat{C F}(Y)$ as a $\mathbb{Z}_{2}[U]$ module where the action of $U$ is multiplication by 0 .

${ }^{4}$ In [10], the authors define a homotopy equivalence between these two chain complexes in a slightly different and more direct way. However, the map $f$ defined here is better suited for our purposes.
} 
4.2. $\mathrm{HF}^{+}$and the contact invariant. Let $\phi$ denote the Whitney triangle between $\overline{x_{g}}, \overline{x_{h}}$, and $\overline{x_{h g}}$ found in Section 3 , and let $\mathfrak{s}(\phi)$ denote the $\operatorname{spin}^{c}$ structure on $X_{g, h}$ corresponding to $\phi$. Moreover, let $\mathfrak{s}_{g}, \mathfrak{s}_{h}$, and $\mathfrak{s}_{h g}$ denote the induced $\operatorname{spin}^{c}$ structures on $Y_{g}, Y_{h}$, and $Y_{h g}$. Then there is a chain map

$$
m_{\mathfrak{s}(\phi)}: \widehat{C F}\left(Y_{g}, \mathfrak{s}_{g}\right) \otimes_{\mathbb{Z}_{2}} \widehat{C F}\left(Y_{h}, \mathfrak{s}_{h}\right) \rightarrow \widehat{C F}\left(Y_{h g}, \mathfrak{s}_{h g}\right)
$$

which is a refinement of the map $m$ defined in Section 3. The difference between the two is that $m_{\mathfrak{s}(\phi)}$ counts only those Whitney triangles which correspond to the spin $^{c}$ structure $\mathfrak{s}(\phi)$. Applying the $\operatorname{Hom}_{\mathbb{Z}_{2}[U]}\left(-, \mathbb{Z}_{2}\left[U, U^{-1}\right] / \mathbb{Z}_{2}[U]\right)$ functor as before and taking homology, it is clear that the induced map

$$
\left(m_{\mathfrak{s}(\phi)}^{\vee}\right)_{*}: \widehat{H F}\left(-Y_{h g}, \mathfrak{s}_{h g}\right) \rightarrow \widehat{H F}\left(-Y_{g}, \mathfrak{s}_{g}\right) \otimes_{\mathbb{Z}_{2}} \widehat{H F}\left(-Y_{h}, \mathfrak{s}_{h}\right)
$$

still takes $c(S, h g) \mapsto c(S, g) \otimes c(S, h)$. Mirroring the notation in Section 3, we denote this map by $\tilde{\mu}_{\mathfrak{s}(\phi)}$.

The pointed Heegaard triple-diagram $(\Sigma, \bar{\alpha}, \bar{\beta}, \bar{\gamma}, z)$ from Section 3 also gives a $U$ equivariant chain map

$$
m_{\mathfrak{s}(\phi)}^{\leq 0}: C F^{\leq 0}\left(Y_{g}, \mathfrak{s}_{g}\right) \otimes_{\mathbb{Z}_{2}[U]} C F^{\leq 0}\left(Y_{h}, \mathfrak{s}_{h}\right) \rightarrow C F^{\leq 0}\left(Y_{h g}, \mathfrak{s}_{h g}\right) .^{5}
$$

Let

$$
\pi_{g \otimes h}: C F^{\leq 0}\left(Y_{g}, \mathfrak{s}_{g}\right) \otimes_{\mathbb{Z}_{2}[U]} C F^{\leq 0}\left(Y_{h}, \mathfrak{s}_{h}\right) \rightarrow \widehat{C F}\left(Y_{g}, \mathfrak{s}_{g}\right) \otimes_{\mathbb{Z}_{2}} \widehat{C F}\left(Y_{h}, \mathfrak{s}_{h}\right)
$$

and

$$
\pi_{h g}: C F^{\leq 0}\left(Y_{h g}, \mathfrak{s}_{h g}\right) \rightarrow \widehat{C F}\left(Y_{h g}, \mathfrak{s}_{h g}\right)
$$

denote the quotient maps discussed in Subsection 4.1. Then the following diagram commutes.

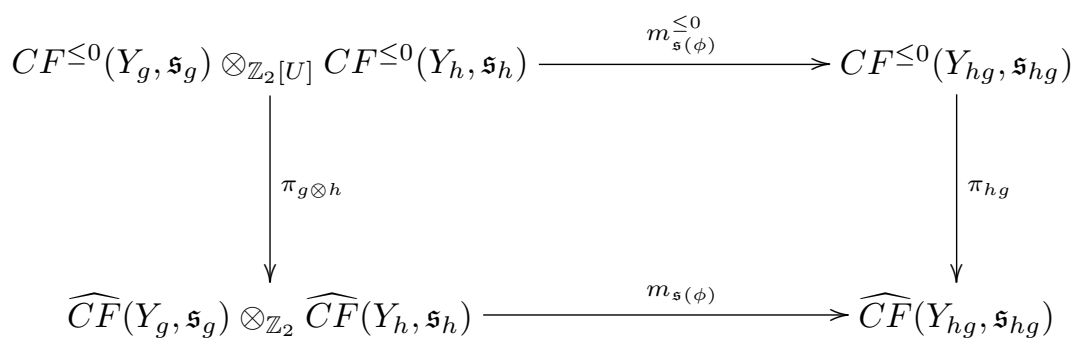

After applying the $\operatorname{Hom}_{\mathbb{Z}_{2}[U]}\left(-, \mathbb{Z}_{2}\left[U, U^{-1}\right] / \mathbb{Z}_{2}[U]\right)$ functor and taking homology, we obtain the commutative diagram

\footnotetext{
${ }^{5}$ For this map to be well-defined, we need $(\Sigma, \bar{\alpha}, \bar{\beta}, \bar{\gamma}, z)$ to be strongly $\mathfrak{s}(\phi)$-admissible. We can assume, however, that this is the case since the Heegaard diagram $(\Sigma, \bar{\alpha}, \bar{\beta}, \bar{\gamma}, z)$ can be made strongly $\mathfrak{s}(\phi)$-admissible by winding the $\bar{\alpha}, \bar{\beta}$, and $\bar{\gamma}$ circles around curves contained strictly in the $-S_{0}$ portion of the surface $\Sigma[11]$.
} 


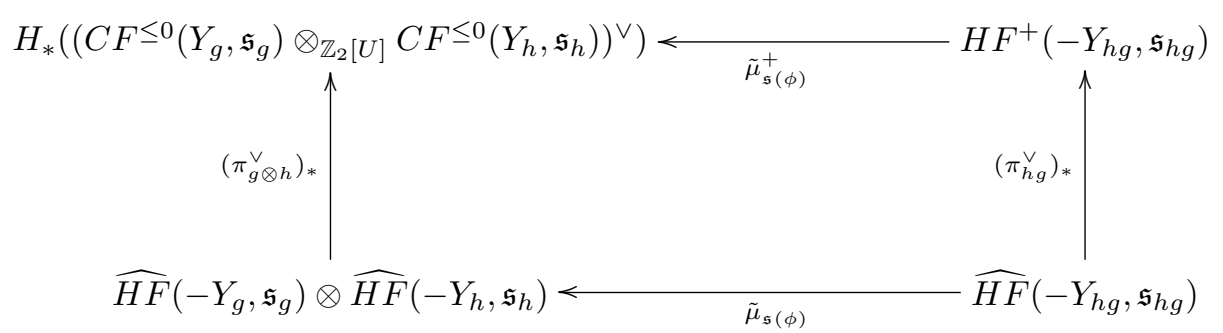

where $\tilde{\mu}_{\mathfrak{s}(\phi)}^{+}=\left(\left(m_{\mathfrak{s}(\phi)}^{\leq 0}\right)^{\vee}\right)_{*}$. Recall that for a contact 3-manifold $(Y, \xi)$, the class $c^{+}(\xi) \in H F^{+}(-Y)$ is defined to be the image of $c(\xi)$ under the natural map $\widehat{H F}(-Y)$ $\rightarrow H F^{+}(-Y)$ [13]. As was mentioned in Subsection 4.1, $\left(\pi_{h g}^{\vee}\right)_{*}$ is this natural map, and therefore

$$
\left(\pi_{h g}^{\vee}\right)_{*}(c(S, h g))=c^{+}(S, h g) .
$$

Let $K_{g \otimes h}$ denote $\tilde{\mu}_{\mathfrak{s}(\phi)}^{+}\left(c^{+}(S, h g)\right)$. Then by the commutativity of this diagram,

$$
K_{g \otimes h}=\tilde{\mu}_{\mathfrak{s}(\phi)}^{+}\left(c^{+}(S, h g)\right)=\left(\pi_{g \otimes h}^{\vee}\right)_{*}(c(S, g) \otimes c(S, h)) .
$$

Returning to our discussion of connected sums, let

$$
\pi_{g \# h}: C F^{\leq 0}\left(Y_{g} \# Y_{h}, \mathfrak{s}_{g} \# \mathfrak{s}_{h}\right) \rightarrow \widehat{C F}\left(Y_{g} \# Y_{h}, \mathfrak{s}_{g} \# \mathfrak{s}_{g}\right)
$$

denote the natural quotient map, and let $f \leq 0$ and $f$ be the homotopy equivalences described in Subsection 4.1. Then the diagram below commutes.

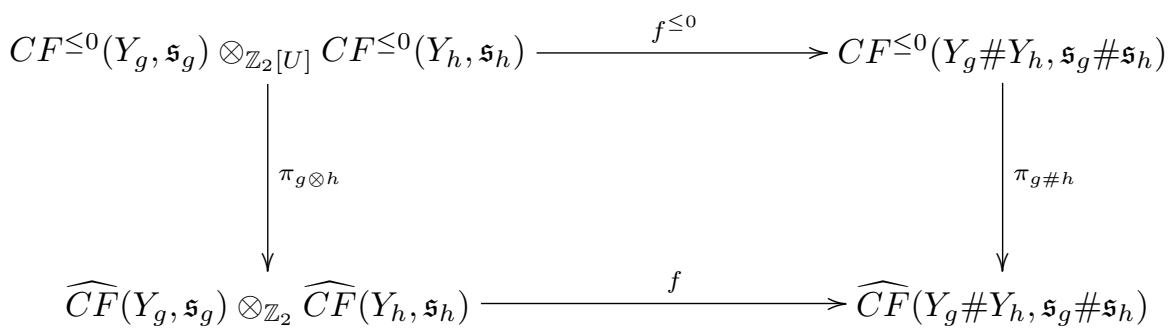

Again, after applying the $\operatorname{Hom}_{\mathbb{Z}_{2}[U]}\left(-, \mathbb{Z}_{2}\left[U, U^{-1}\right] / \mathbb{Z}_{2}[U]\right)$ functor and taking homology, we obtain the commutative diagram

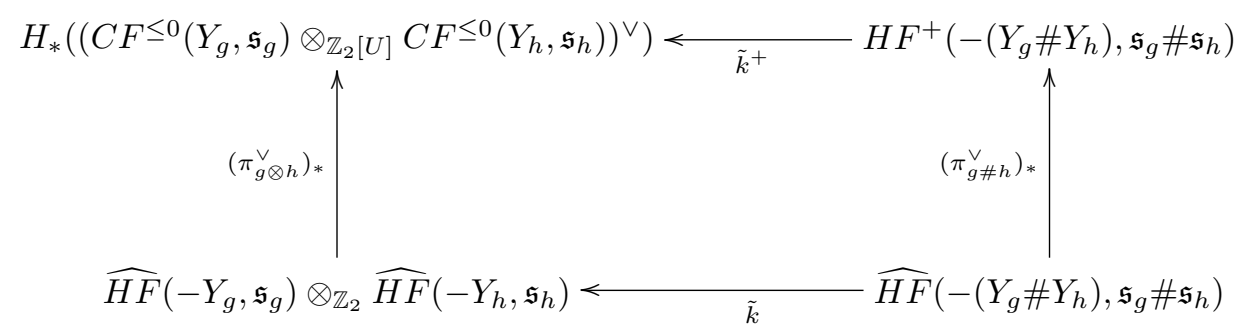

where $\tilde{k}^{+}=\left(\left(f^{\leq 0}\right)^{\vee}\right)_{*}$ and $\tilde{k}=\left(f^{\vee}\right)_{*}$. At this point, we are ready to generalize Theorem 1.4. 
Theorem 4.1. The map $\left(\tilde{k}^{+}\right)^{-1} \circ \tilde{\mu}_{\mathfrak{s}(\phi)}^{+}: H F^{+}\left(-Y_{h g}, \mathfrak{s}_{h g}\right) \rightarrow$ $H F^{+}\left(-\left(Y_{g} \# Y_{h}\right), \mathfrak{s}_{g} \# \mathfrak{s}_{h}\right)$ is $U$-equivariant and takes $c^{+}(S, h g) \mapsto c^{+}\left(S \#{ }_{b} S, g \# h\right)$.

Observe that Theorem 1.5 follows immediately as a corollary. Recall from the introduction that the contact structure compatible with the open book $\left(S \#_{b} S, g \# h\right)$ is the connected sum of the contact structures compatible with $(S, g)$ and $(S, h)$.

Proof of Theorem 4.1. The maps $\tilde{\mu}_{\mathfrak{s}(\phi)}^{+}$and $\left(\tilde{k}^{+}\right)^{-1}$ are certainly $U$-equivariant, so their composition is as well. Moreover, $\tilde{k}=\left(f^{\vee}\right)_{*}$ takes $c\left(S \#_{b} S, g \# h\right) \mapsto c(S, g) \otimes$ $c(S, h)$. This follows from precisely the same sort of argument as was used in Section 3 to show that $\tilde{\mu}=\left(m^{\vee}\right)_{*}$ takes $c(S, h g) \mapsto c(S, g) \otimes c(S, h)$. In the pointed Heegaard triple-diagram $\left(\Sigma_{1} \# \Sigma_{2}, \bar{\alpha}_{1} \cup \bar{\alpha}_{2}^{\prime}, \bar{\beta}_{1} \cup \bar{\alpha}_{2}, \bar{\beta}_{1}^{\prime} \cup \bar{\beta}_{2}, z\right)$ used to construct the map $\Gamma$, the only holomorphic Whitney triangle $\phi \in \pi_{2}\left(\bar{a}, \bar{b}, \overline{x_{g}} \times \overline{x_{h}}\right)$ with $n_{z}(\phi)=0$ is a product of small triangles connecting $\overline{x_{g}} \times \Theta_{2}, \Theta_{1} \times \overline{x_{h}}$, and $\overline{x_{g}} \times \overline{x_{h}}$. Therefore, the map $f^{\vee}$ takes $\overline{x_{g}} \times \overline{x_{h}} \mapsto \overline{x_{g}} \otimes \overline{x_{h}}$ on the level of chains, and $\left(f^{\vee}\right)_{*}$ takes $c\left(S \#{ }_{b} S, g \# h\right) \mapsto$ $c(S, g) \otimes c(S, h)$.

Hence,

$$
\left(\left(\pi_{g \otimes h}^{\vee}\right)_{*} \circ \tilde{k}\right)\left(c\left(S \#{ }_{b} S, g \# h\right)\right)=\left(\pi_{g \otimes h}^{\vee}\right)_{*}(c(S, g) \otimes c(S, h))=K_{g \otimes h} .
$$

Therefore, by commutativity,

$$
K_{g \otimes h}=\left(\tilde{k}^{+} \circ\left(\pi_{g \# h}^{\vee}\right)_{*}\right)\left(c\left(S \#_{b} S, g \# h\right)\right)=\tilde{k}^{+}\left(c^{+}\left(S \#{ }_{b} S, g \# h\right)\right) .
$$

Thus, $\left(\tilde{k}^{+}\right)^{-1}\left(K_{g \otimes h}\right)=c^{+}\left(S \#_{b} S, g \# h\right)$. But this implies that

$$
\left(\left(\tilde{k}^{+}\right)^{-1} \circ \tilde{\mu}_{\mathfrak{s}(\phi)}^{+}\right)\left(c^{+}(S, h g)\right)=\left(\tilde{k}^{+}\right)^{-1}\left(K_{g \otimes h}\right)=c^{+}\left(S \#{ }_{b} S, g \# h\right) .
$$

\section{5. $L$-spaces and planar open books}

Etnyre recently showed that while every overtwisted contact structure has a compatible open book with planar pages, there are fillable contact structures which do not [2]. More recently, Ozsváth, Szabó, and Stipsicz found a Heegaard Floer homology obstruction to a contact structure having a compatible open book with planar pages, and were able to reproduce some of Etnyre's results [13]. Their main result is the following:

Theorem 5.1 (Ozsváth-Szabó-Stipsicz). If the contact 3-manifold $(Y, \xi)$ has a compatible open book with planar pages then $c^{+}(\xi) \in U^{d} \cdot H F^{+}(-Y)$ for all $d \in \mathbb{N}$.

They prove the following corollaries which are based upon this principle.

Corollary 5.2 (Ozsváth-Szabó-Stipsicz). Suppose that $c^{+}(\xi) \neq 0$ and $c_{1}(\mathfrak{s}(\xi)$ ) is non-torsion. Then it cannot be the case that $c^{+}(\xi) \in U^{d} \cdot H F^{+}(-Y)$ for all $d \in \mathbb{N}$. In particular, $(Y, \xi)$ is not supported by a planar open book.

Corollary 5.3 (Ozsváth-Szabó-Stipsicz). Suppose that the contact 3-manifold $(Y, \xi)$ has a Stein filling $(X, J)$ with $c_{1}(\mathfrak{s}(\xi))=0$ and $c_{1}(X, J) \neq 0$. Then it cannot be the case that $c^{+}(\xi) \in U^{d} \cdot H F^{+}(-Y)$ for all $d \in \mathbb{N}$. In particular, $(Y, \xi)$ is not supported by a planar open book.

Our Corollaries 1.6 and 1.7 now follow from Theorems 1.5, 4.1, and the above corollaries of Ozsváth, Szabó, and Stipsicz. 
Proof of Corollary 1.6. If $c_{1}(\mathfrak{s}(S, g))$ is non-torsion, then so is

$$
c_{1}\left(\mathfrak{s}\left(S \#{ }_{b} S, g \# h\right)\right)=c_{1}(\mathfrak{s}(S, g)) \oplus c_{1}(\mathfrak{s}(S, h)) .
$$

If, in addition, $c^{+}\left(S \#_{b} S, g \# h\right) \neq 0$, then Corollary 5.2 implies that it cannot be the case that $c^{+}\left(S \#{ }_{b} S, g \# h\right) \in U^{d} \cdot H F^{+}\left(-\left(Y_{g} \# Y_{h}\right)\right)$ for all $d \in \mathbb{N}$. Thus, Theorem 1.5 implies that it cannot be the case that $c^{+}(S, h g) \in U^{d} \cdot H F^{+}\left(-Y_{h g}\right)$ for all $d \in \mathbb{N}$. It follows that $Y_{h g}$ cannot be an $L$-space. Furthermore, it follows from Theorem 5.1 of Oszváth, Szabó, and Stipsicz that the contact structure supported by $(S, h g)$ is not compatible with a planar open book.

Proof of Corollary 1.7. If $(S, g)$ and $(S, h)$ correspond to contact manifolds $\left(Y_{g}, \xi_{g}\right)$ and $\left(Y_{h}, \xi_{h}\right)$ with Stein fillings $\left(X_{g}, J_{g}\right)$ and $\left(X_{h}, J_{h}\right)$, then $\left(S \#_{b} S, g \# h\right)$ corresponds to the contact manifold $\left(Y_{g} \# Y_{h}, \xi_{g} \# \xi_{h}\right)$ with Stein filling $\left(X_{g} \#_{b} X_{h}, J_{g} \#_{b} J_{h}\right)$. If $c_{1}\left(\mathfrak{s}\left(\xi_{g}\right)\right)=0$ and $c_{1}\left(\mathfrak{s}\left(\xi_{h}\right)\right)=0$, then

$$
c_{1}\left(\mathfrak{s}\left(\xi_{g} \# \xi_{h}\right)\right)=c_{1}\left(\mathfrak{s}\left(\xi_{g}\right)\right) \oplus c_{1}\left(\mathfrak{s}\left(\xi_{h}\right)\right)=0 .
$$

Moreover, if $c_{1}\left(X_{g}, J_{g}\right) \neq 0$, then

$$
c_{1}\left(X_{g} \#_{b} X_{h}, J_{g} \#_{b} J_{h}\right)=c_{1}\left(X_{g}, J_{g}\right) \oplus c_{1}\left(X_{h}, J_{h}\right) \neq 0 .
$$

Then, by Corollary 5.3, it cannot be the case that $c^{+}\left(S \#_{b} S, g \# h\right) \in$ $U^{d} \cdot H F^{+}\left(-\left(Y_{g} \# Y_{h}\right)\right)$ for all $d \in \mathbb{N}$. And, just as before, Theorem 1.5 then implies that it cannot be the case that $c^{+}(S, h g) \in U^{d} \cdot H F^{+}\left(-Y_{h g}\right)$ for all $d \in \mathbb{N}$. It follows that $Y_{h g}$ cannot be an $L$-space and that the contact structure supported by $(S, h g)$ is not compatible with a planar open book.

It is not clear whether we can replace the condition that $c^{+}\left(S \#_{b} S, g \# h\right) \neq 0$ in the formulation of Corollary 1.6 by the condition that $c^{+}(S, g) \neq 0$ and $c^{+}(S, h) \neq 0$. For the contact invariant $c$ defined in $\widehat{H F}(-Y), c(S, g) \neq 0$ and $c(S, h) \neq 0$ implies that $c\left(S \#_{b} S, g \# h\right) \neq 0$. It is not immediately obvious that the same is true in general for the contact invariant $c^{+}$. There are, however, special cases in which the same holds for $c^{+}$. For instance, if $(S, g)$ and $(S, h)$ support strongly fillable contact structures, then so does $\left(S \#_{b} S, g \# h\right)$, and hence, $c^{+}\left(S \#_{b} S, g \# h\right) \neq 0$. More useful perhaps, is the following.

Claim 5.4. If $c^{+}(S, g) \neq 0$ and $(S, h)$ supports a Stein fillable contact structure, then $c^{+}\left(S \#_{b} S, g \# h\right) \neq 0$.

Proof of Claim 5.4. If $(S, h)$ supports a Stein fillable contact structure, then after a number of positive stabilizations $(S, h)$ is equivalent to an open book $\left(S^{\prime}, \phi\right)$, where $\phi$ is the composition of right-handed Dehn twists around curves in $S^{\prime}$. Hence, $\left(S \#_{b} S, g \# h\right)$ is equivalent via the same number of positive stabilizations to the open book $\left(S \#_{b} S^{\prime}, g \# \phi\right)$. Therefore, by the naturality of the contact invariant $c^{+}$under composition with left-handed Dehn twists, if $c^{+}\left(S \#_{b} S^{\prime}, g \# i d\right) \neq 0$, then $c^{+}\left(S \#_{b} S^{\prime}, g \# \phi\right) \neq 0$. ${ }^{6}$ Let $m=2 k+r$, where $k$ is the genus of $S^{\prime}$ and $r$ is the number of boundary components of $S^{\prime}$. Then $\left(S \#_{b} S^{\prime}, g \# i d\right)$ is an open book for the manifold $Y_{g} \# \#^{m}\left(S^{1} \times S^{2}\right)$.

\footnotetext{
${ }^{6}$ Theorem 1.4 was stated only for the contact invariant $c$, but the same holds for $c^{+}$via a commutative diagram chase.
} 
There is an isomorphism

$$
H F^{+}\left(-\left(Y_{g} \# \#^{m}\left(S^{1} \times S^{2}\right)\right), \mathfrak{s}_{g} \# \mathfrak{s}_{0}\right) \rightarrow H F^{+}\left(-Y_{g}, \mathfrak{s}_{g}\right) \otimes \wedge^{*} H^{1}\left(\#^{m}\left(S^{1} \times S^{2}\right)\right)
$$

[10, Proposition 6.4]. It is clear from the construction that this isomorphism takes

$$
c^{+}\left(S \#_{b} S^{\prime}, g \# i d\right) \mapsto c^{+}(S, g) \otimes \theta
$$

where $\theta$ is the lowest graded element of $\wedge^{*} H^{1}\left(\#^{m}\left(S^{1} \times S^{2}\right)\right)$. Therefore, $c^{+}(S, g) \neq 0$ if and only if $c^{+}\left(S \#_{b} S^{\prime}, g \# i d\right) \neq 0$. Putting these facts together, we get the claim.

It remains to be seen what can be shown using these techniques. It would be very interesting, as Etnyre mentions in [2], to find a non-fillable contact structure which is not supported by an open book with planar pages. To this end it is enough to find, by Corollary 1.6, a Stein fillable open book $(S, g)$ with $c_{1}(\mathfrak{s}(S, g))$ non-torsion and an open book $(S, h)$ with $c^{+}(S, h) \neq 0$ such that $(S, h g)$ is non-fillable.

\section{Acknowledgements}

I would like to thank Ko Honda for bringing to my attention the possibility of Theorem 1.2. I also wish to thank John Etnyre, Danny Gillam, Robert Lipshitz, and Shaffiq Welji for very helpful discussions. And, as always, I am indebted to Peter Ozsváth for his invaluable comments and suggestions.

\section{References}

[1] J. A. Baldwin, Tight contact structures and genus one fibered knots, Algebr. Geom. Topol. 7 (2007), 701-735.

[2] J. B. Etnyre, Planar open book decompositions and contact structures, Int. Math. Res. Not. 79 (2004), 4255-4267.

[3] Lectures on open book decompositions and contact structures, Clay Math. Proc., 5, Amer. Math. Soc., Providence, RI, 2006.

[4] P. Ghiggini, Ozsváth-Szabó invariants and fillability of contact structures, Math. Z. 253 (2006), no. 1, 159-175.

[5] P. Ghiggini, K. Honda, and J. Van Horn-Morris, The vanishing of the contact invariant in the presence of torsion, 2007, math.GT/0706.1602.

[6] E. Giroux, Convexité en topologie de contact, Comment. Math. Helv. 66 (1991), 637-677.

[7] K. Honda, W. Kazez, and G. Matić, On the contact class in Heegaard Floer homology, 2006, math.GT/0609734.

[8] _ Right-veering diffeomorphisms of a compact surface with boundary II, 2006, math.GT/0603626.

[9] _ Right-veering diffeomorphisms of a compact surface with boundary, Inv. Math. 169 (2007), no. 2, 427-449.

[10] P. Ozsváth and Z. Szabó, Holomorphic disks and three-manifold invariants: properties and applications, Annals of Mathematics, 159 (2004), no. 3, 1159-1245.

[11] Holomorphic disks and topological invariants for closed three-manifolds, Annals of Mathematics, 159 (2004), no. 3, 1027-1158.

[12] Heegaard Floer homologies and contact structures, Duke Math. J. 129 (2005), no. 1, 39-61.

[13] P. Ozsváth, Z. Szabó, and A. I. Stipsicz. Planar open books and Floer homology, Int. Math. Res. Not. (2005), no. 54, 3385-3401.

Department of Mathematics, Columbia University, New York, NY 10027

E-mail address: baldwin@math.columbia.edu 